\title{
Artigo original \\ Experiência subjetiva de idosas durante exercício em ambiente virtual
}

\author{
Amanda Mayara do Nascimento \\ Nara Heloisa Rodrigues \\ Universidade Estadual Paulista \\ Eric Francelino Andrade \\ Gustavo Puggina Rogatto \\ Universidade Federal de Lavras \\ Gisele Maria Schwartz \\ Universidade Estadual Paulista \\ Priscila Carneiro Valim-Rogatto \\ Universidade Federal de Lavras
}

\begin{abstract}
Resumo-Os objetivos desse estudo quali-quantitativo foram verificar as experiências subjetivas relacionadas ao exercício em idosas antes e depois da realização de atividade física em ambientes real e virtual, bem como a

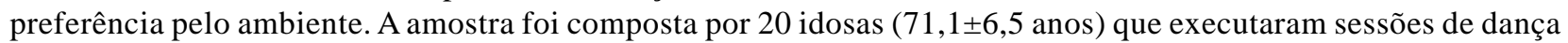
nos dois ambientes e responderam ao instrumento Subjective Exercise Experiences Scale (SEES) antes e depois da prática. Na situação virtual foi utilizado o jogo Just Dance I do console Wii ${ }^{\circledR}$ da Nintendo. Na situação real, cada participante reproduziu os mesmos movimentos demonstrados pela pesquisadora. Para a análise dos dados da SEES foram utilizados os testes Wilcoxon e Mann-Whitney $(p<0,05)$. Houve redução significativa na pontuação da subescala distress psicológico da SEES após ambas as situações experimentais e aumento no bem-estar positivo apenas em ambiente virtual. A maioria das idosas escolheu o ambiente real como o preferido.
\end{abstract}

Palavras-chaves: exercício, idoso, tecnologia, comportamento

Abstract-“Subjective experience of older women during exercise in virtual condition.” This quali-quantitative study aimed to verify subjective exercise experiences of older women before and after exercise sessions in actual and virtual conditions, as well as, the preferred exercise condition. The sample was composed by 20 older women (71.1 \pm 6.5 years-old) who practiced dance sessions in actual and virtual conditions, and answered the Subjective Exercise Experiences Scale (SEES) before and after the activity. In virtual condition it was used the virtual game Just Dance I from the Nintendo Wii ${ }^{\circledR}$ console. In actual condition participants reproduced the same game movements, as demonstrated by the researcher. Wilcoxon and Mann-Whitney tests were used for data analyses $(p<0.05)$. Significant decrease was observed in the psychological distress score of SEES after both conditions. Increase in positive well being score was found only in the virtual condition. Most of the older women chose the actual condition as the preferred.

Keywords: exercise, aging, technology, behavior

Resumen-“Experiencia subjetiva de mujeres ancianas durante el ejercicio en el ambiente virtual.” Los objetivos de este estudio cuali-cuantitativo fueron evaluar las experiencias subjetivas relacionadas con el ejercicio en mujeres ancianas antes y después de la actividad física en ambientes real y virtual, así como la preferencia por el ambiente. La muestra consistió en 20 mujeres ancianas (71,1 \pm 6,5 años) que ejecutaran sesiones de danza en ambos ambientes y responderán al instrumento Subjective Exercise Experiences Scale (SEES) antes y después de la práctica. En la situación virtual fue empleado el juego Just Dance I de la consola Nintendo ${ }^{\circledR}$ Wii. En la situación real, cada participante reprodujo los mismos movimientos presentados por el investigador. Para el análisis de los datos de la SEES se utilizó las pruebas de Wilcoxon y Mann-Whitney $(p<0,05)$. Hubo reducción significativa en la puntuación de la subescala distress psicológico de la SEES después de ambas las situaciones experimentales y aumento del bienestar positivo sólo en el ambiente virtual. La mayoría de las mujeres eligieron el ambiente real como el favorito.

Palabras claves: ejercicio, anciano, tecnología, conducta 


\section{Introdução}

O interesse em estudos relacionados aos estados afetivos e respostas psicológicas relacionadas ao exercício físico em idosos tem se intensificado nos últimos anos. Focht, Knapp, Gavin, Raedeke, e Hickner (2007) afirmam que ao experimentar respostas psicológicas favoráveis no exercício agudo, o idoso pode apresentar maior probabilidade de se envolver em programas de exercícios regulares no futuro.

Estudos têm evidenciado que declínios físicos e comprometimentos psicológicos e sociais, decorrentes do processo de envelhecimento, podem ser retardados quando os idosos são praticantes ativos de exercícios físicos (Nelson et al., 2007). Um conjunto considerável de pesquisas tem demonstrado que episódios únicos de exercícios aeróbios podem induzir mudanças em uma variedade de manifestações de estados afetivos como, por exemplo, melhoria nos estados de humor (Berger \& Motl, 2000; Krug, Conceição, Mazo, Antunes, \& Romitti, 2011) e alterações positivas em relação ao estresse e à ansiedade (Focht et al., 2007). Evidências crescentes também têm confirmado melhoria do bem-estar psicológico em idosos após a participação regular por longo tempo em programas de exercícios (McAuley, Elsavsky, Jerome, Konopack, \& Marquez, 2005).

Segundo Hoffman e Harris (2002), os efeitos psicológicos do exercício podem estar associados à prevenção dos transtornos da saúde mental devido à força das evidências demonstradas em sessões agudas (curto prazo) de exercício que podem reduzir a ansiedade; e em atividades crônicas (exercício regular por um período de tempo prolongado) que podem reduzir os riscos de ocorrência de depressão. A atividade física regular pode estar relacionada com a melhora da sensação de bem-estar psicológico devido ao aumento das secreções hormonais que provocam alterações no estado de humor e/ou reduzem as reações ao estresse e à ansiedade (Sciolino \& Holmes, 2012).

Entretanto, alterações nos estados psicológicos após exercício agudo em idosos ainda precisam ser mais investigadas, pois estudos nessa temática ainda não deixaram claro se as experiências afetivas relacionadas a este tipo de exercício apresentam benefícios similares com aquelas documentadas envolvendo participantes mais jovens (Focht et al., 2007).

Com o notável aumento da expectativa de vida dos idosos, tem-se observado maior acesso dos mesmos às novas tecnologias e jogos eletrônicos atuais como, por exemplo, os videogames. Os idosos estão se mostrando cada vez mais interessados na utilização de aparatos tecnológicos como meio de interação com a sociedade atual e para obtenção de maior sensação de bem-estar, saúde e qualidade de vida (Allaire, McLaughlin, Trujillo, Whitlock, LaPorte, \& Gangy, 2013). Além disso, trabalhos nessa temática evidenciam que existem alguns modelos de videogames ativos que estão sendo usados justamente por atender melhor às necessidades da população idosa, como é o caso do console Wii da Nintendo ${ }^{\circledR}$ (Schiesel, 2007; Rosemberg et al., 2010).

Os videogames ativos podem ser caracterizados como sendo os jogos baseados nos movimentos de diversos segmentos corporais como forma de interação com ambientes virtuais, que podem contribuir tanto para o aumento do gasto calórico (Haddock et al., 2012) quanto para o aumento no repertório de movimentos do sujeito. A literatura científica tem trabalhado com diferentes terminologias para se referir a essa nova forma de interação, tais como exergames, videojogos, exergaming, motion-sensing video game, activity-promoting video games, entre outras (Allaire et al., 2013; Baracho, Gripp, \& Lima, 2012; Tanaka, Parker, Baradoy, Sheehan, Holash, \& Katz, 2012; Vaghetti \& Botelho, 2010). A partir da necessidade de sistematização de apenas uma terminologia para ser utilizada no desenvolvimento do presente estudo, optou-se pelo termo videogames ativos.

Com o crescimento da indústria de jogos eletrônicos, o uso de videogames ativos tem se apresentado para o idoso como uma interessante alternativa para a prática de exercícios de maneira lúdica e prazerosa. Nesse sentido, pesquisas reforçam a influência positiva da utilização de videogames pela população idosa como meio de entretenimento, satisfação e bem-estar (Torres \& Zagalo, 2007).

$\mathrm{Na}$ literatura, encontram-se estudos (embora escassos) realizados por pesquisadores que lançam mão de delineamentos que incluem a utilização do videogame ativo Wii ${ }^{\circledR}$ como instrumento para a prática de exercício físico com a participação da população idosa (Rosemberg et al., 2010; Torres \& Zagalo, 2007; Williams, Soiza, Jenkinson, \& Stewart, 2010). O Wii da Nintendo ${ }^{\circledR}$ caracteriza-se por ser um console de videogame doméstico onde a principal ação é a utilização de dispositivos sem fio para detectar os movimentos do usuário e, dessa forma, poder obter o controle sobre o jogo. O Wii remote ${ }^{\circledR}$ é um controle que possui um sensor de movimentos que capta pontos de luz infravermelhos transmitidos por um emissor (sensor bar) geralmente situado sobre ou sob um aparelho de televisão. Assim, é possível perceber para qual ponto da tela da televisão se está apontando o controle (Wii Consoles, 2009). Essas características específicas possibilitam que os usuários não precisem se restringir à manipulação de botões ou joysticks para controlar os seus jogos. Com o Wii ${ }^{\circledR}$ tornou-se possível movimentar, apontar, inclinar e balançar o controlador, o que abriu uma nova perspectiva de utilização do game para a prática de jogos baseados em esportes como, por exemplo, o golfe e o tênis. Além disso, no controle há uma pequena caixa de som que permite que as ondas sonoras cheguem melhor e mais perto do jogador, ampliando o realismo dos jogos.

Partindo desses pressupostos, é possível constatar que a prática de exercício físico com a utilização de videogames ativos, como o Nintendo $\mathrm{Wii}^{\circledR}$, associada às respostas afetivas e capacidades físicas em idosos começa a ser investigada, porém, diversos aspectos ainda estão inexplorados. Dessa forma, torna-se necessário que novas pesquisas sejam conduzidas com o intuito de subsidiar essa temática, na tentativa de ampliar as possibilidades de formulação de novas estratégias que possam contribuir para a adesão e manutenção da prática de exercício no ambiente virtual por esse grupo etário. 
Sendo assim, os objetivos do presente estudo foram verificar as experiências subjetivas relacionadas ao exercício de idosas antes e depois da prática de exercício nos ambientes real e virtual e verificar a preferência pelo ambiente de exercício.

\section{Método}

A amostra do tipo não-probabilística foi composta por 20 idosas com média de idade de $71,1( \pm 6,5)$ anos, residentes na cidade de Lavras, situada no sul do Estado de Minas Gerais. A coleta de dados foi realizada no Laboratório de Pesquisa em Psicologia do Exercício (LAPPEX) localizado no Departamento de Educação Física da Universidade Federal de Lavras (UFLA). As participantes da amostra foram informadas da pesquisa por meio de contato pessoal com os pesquisadores. Nesse contato, foram explicitados os objetivos pretendidos, bem como esclarecidos os conteúdos a serem investigados. Todas as voluntárias assinaram o Termo de Consentimento Livre e Esclarecido (TCLE) para a participação na pesquisa. O presente estudo é parte do projeto de pesquisa intitulado "Exergames: aspectos psicológicos e fisiológicos relacionados à experiência de prática de exercícios em ambiente virtual" devidamente aprovado pelo Comitê de Ética em Pesquisa com Seres Humanos da Universidade Federal de Lavras, sob o protocolo CAAE - 0010.0.461.000-11.

Inicialmente cada participante respondeu a dois Questionários: 1) Questionário para a caracterização da amostra contendo informações sobre idade, sexo, experiência do indivíduo em determinadas atividades físicas como, dança e/ou ginástica e também informações acerca do contato com algum tipo de videogame; 2) Questionário Internacional de Atividade Física- IPAQ versão curta (Matsudo, Matsudo, Araújo, Andrade,\& Oliveira, 2002), que é um instrumento que estima o nível de atividade física por meio de informações sobre frequência (dias por semana) e duração (minutos por dia) da realização de atividades físicas moderadas, vigorosas e caminhada em uma semana habitual. Em conformidade com o guia de processamento de dados e análises do IPAQ (Guidelines, 2008) foram seguidos os procedimentos necessários para o cálculo de escores para a classificação dos indivíduos em três categorias de nível de atividade física: Baixo, Moderado e Alto. Todos os questionários foram aplicados individualmente.

As avaliações físicas às quais as participantes foram submetidas durante o estudo foram: massa (Kg) e estatura (cm) aferidas com a utilização de uma balança digital com estadiômetro da marca Welmy, modelo W 200/5. A partir das medidas obtidas, foi calculado o índice de massa corporal $(\mathrm{IMC})=$ massa dividida pela estatura ao quadrado $\left(\mathrm{Kg} / \mathrm{m}^{2}\right)$ (WHO, 1995).

Foi utilizada a tradução proposta por Miranda (2001) do instrumento Subjective Exercise Experiences Scale - SEES (McAuley \& Courneya, 1994) para avaliar os estados afetivos, ou seja, as experiências subjetivas relacionadas ao exercício antes e depois desta prática em ambos os ambientes (real e virtual). A SEES é constituída de três subescalas objetivas que somam 12 adjetivos no total. A subescala bemestar positivo é composta pelos seguintes adjetivos: ótimo, positivo, vigoroso e maravilhoso; a subescala distress psicológico é composta pelos adjetivos péssimo, diminuído, desanimado e triste; enquanto na subescala fadiga, os adjetivos são: esgotado, exausto, fatigado e cansado. Cada adjetivo pode ser classificado em determinadas intensidades que correspondem a pontuações que podem variar de 1 (que significa "nada") a 7 pontos (que significa "muito"). Dessa forma, a pontuação em cada subescala pode ser de no mínimo 4 e de no máximo 28 pontos. Assim, quanto maior a pontuação na subescala bem-estar positivo, maiores são os benefícios experimentados. Maiores pontuações nas subescalas distress psicológico e fadiga indicam fortes associações relacionadas a fatores negativos ou desagradáveis experimentados pelo indivíduo em exercício.

As voluntárias participaram individualmente de duas sessões de exercício agudo: sessão em ambiente real e sessão em ambiente virtual. A ordem de execução das sessões foi definida aleatoriamente para cada voluntária, para que a sequência utilizada não interferisse nos resultados da pesquisa. As sessões ocorreram com o mínimo de 48 horas de intervalo para que não existisse interferência dos efeitos de uma sessão em outra.

Para a realização da sessão de exercício em ambiente virtual foi utilizado o console Wii da Nintendo ${ }^{\circledR}$. Nesta avaliação, as participantes permaneceram a uma distância de 2,5 metros em relação ao sensor bar (sensor de movimento) e a 3 metros em relação à TV de tela plana de 32 polegadas (distância de 0,5 m entre a televisão e o sensor bar). Estas distâncias foram padronizadas em conformidade com a indicação do Manual do Funcionamento do Videogame que recomenda que haja uma distância de um a três metros do televisor. No presente estudo, foi utilizado o jogo de dança Just Dance I, produzido pela Ubisoft ${ }^{\circledR}$. Neste jogo, os jogadores devem apenas segurar com uma das mãos o Wii remote, sem precisar acionar combinações de botões do controle, e acompanhar os movimentos de um personagem que dança exibido na tela da TV. As voluntárias executaram os movimentos da música Wanna Be - Spice Girls do referido jogo. As participantes reproduziram a coreografia conforme os movimentos conduzidos pela personagem do jogo. A música utilizada tem a duração de 2 minutos e 45 segundos e andamento musical de 110 Batimentos por minuto (Bpm). Durante o tempo dessa música são realizados 13 movimentos de dança/ginástica.

Na sessão de exercício em ambiente real, as voluntárias realizaram os mesmos procedimentos tomados na sessão virtual. Entretanto, os movimentos coreográficos de dança da música supracitada foram demonstrados por uma das pesquisadoras e executados pelas participantes.

A SEES foi aplicada antes e logo depois de cada sessão de exercício. Para a análise da preferência quanto às sessões executadas foi perguntado para cada pessoa após sua última sessão experimental qual dos dois ambientes (virtual ou real) foi o preferido para a prática do exercício. 
A estatística descritiva (média, desvio-padrão e porcentagem, quando aplicáveis) foi utilizada para a caracterização da amostra pesquisada. A verificação da normalidade dos dados referentes à SEES foi realizada pela aplicação do teste Shapiro-Wilk. Como os dados da SEES não apresentaram distribuição normal, optou-se pela análise estatística não-paramétrica para o tratamento dos dados. O teste Wilcoxon e o teste Mann-Whitney foram utilizados para analisar o fator tempo (antes $\mathrm{x}$ depois) e o fator bloco (ambiente real $\mathrm{x}$ ambiente virtual) respectivamente em cada uma das três subescalas da SEES (bem-estar positivo, distress psicológico e fadiga). O teste Wilcoxon é um teste não-paramétrico poderoso para comparar amostras relacionadas com grupos dependentes (pareados) (Thomas \& Nelson, 2002) enquanto que o teste Mann-Whitney é utilizado no caso de comparações entre duas amostras nãopareadas com a finalidade de verificar se dois grupos independentes foram extraídos da mesma população (Siegel, 1975). Para todas as análises, o nível de significância adotado foi de $5 \%(p<0,05)$.

Os dados foram analisados utilizando-se do software livre BioEstat 5.0 (Ayres, Ayres Júnior, Ayres, \& Santos, 2007).

\section{Resultados}

As características físicas das idosas da amostra pesquisada podem ser observadas na Tabela 1.

Quanto ao nível de atividade física das participantes do estudo, 60\% foram classificadas no nível "moderado", 35\% no nível "baixo" e 5\% no nível "alto" de atividade física.

Quanto à experiência em algum tipo de dança e/ou ginástica, 85\% das idosas relataram ter vivenciado algum tipo dessas atividades e $15 \%$ disseram que nunca tiveram contato com nenhuma delas.

Tabela 1. Características físicas da amostra pesquisada $(\mathrm{n}=20)$.

\begin{tabular}{lcc}
\hline & Média & Desvio-Padrão \\
\hline Idade (anos) & 71,1 & 6,5 \\
Estatura (m) & 1,56 & 0,05 \\
Massa $(\mathrm{Kg})$ & 63,8 & 10,4 \\
IMC $\left(\mathrm{Kg} / \mathrm{m}^{2}\right)$ & 25,9 & 3,4 \\
\hline
\end{tabular}

Até o momento da participação na presente pesquisa, nenhuma voluntária relatou ter utilizado qualquer tipo de videogame anteriormente.

Em relação às modificações observadas entre antes e depois da execução dos exercícios, a redução da pontuação na subescala distress psicológico foi verificada tanto no ambiente real quanto no virtual. Houve aumento significativo na pontuação da subescala de bem-estar positivo apenas no ambiente virtual. Não foram encontradas diferenças significativas entre a pontuação da subescala fadiga obtida antes e depois da realização do exercício tanto em ambiente real quanto em ambiente virtual. As pontuações obtidas em cada subescala da SEES em ambientes real e virtual (antes e depois do exercício) podem ser verificadas nas Tabelas 2 e 3 , respectivamente.

Quanto às comparações referentes ao ambiente do exercício, em todas as subescalas da SEES não foram encontradas diferenças significativas entre o ambiente virtual (AV) e ambiente real (AR), conforme pode ser observado na Tabela 4.

Quanto à preferência das idosas em relação ao ambiente da sessão de exercício, 70\% delas relataram ser o AR o preferido para a prática da atividade física pesquisada.

\section{Discussão}

Em relação ao nível de atividade física, as idosas da amostra demonstraram ser mais ativas que os idosos analisados em outros estudos encontrados na literatura (Benedetti, Binotto, Petroski, \& Gonçalves, 2008; Siqueira et al., 2007) uma vez que, $65 \%$ das idosas do presente estudo se encontravam dentro dos níveis de atividade física recomendados pelo Colégio Americano de Medicina do Esporte (soma dos níveis moderado 60\% e alto 5\%) (Nelson et al, 2007). A prevalência de inatividade física em indivíduos idosos no Brasil foi observada em um estudo conduzido por Hallal, Victoria, Wells e Lima (2003). Os autores constataram que $43,2 \%$ das mulheres entrevistadas de 60 a 69 anos eram inativas e que, com o aumento da idade, esses valores aumentaram, atingindo 69,1\% nos indivíduos acima de setenta anos. Em estudo recente realizado por Valim-Rogatto, Candolo e Bretas (2011), com a utilização do IPAQ versão

Tabela 2. Média, desvio-padrão, mediana, desvio interquartílico e resultados do teste Wilcoxon para cada subescala da SEES referentes ao exercício realizado em AR $(n=20)$.

\begin{tabular}{|c|c|c|c|c|c|c|c|c|c|c|}
\hline & & & Antes & & & & & & & \\
\hline SEES & Média & DP & Mediana & DI & Média & DP & Mediana & DI & $\mathrm{z}$ & $p$ \\
\hline BEP & 22,9 & 4,4 & 24,0 & 5,25 & 24,1 & 3,8 & 25,5 & 6,0 & 1,46 & 0,14 \\
\hline DPS & 8,5 & 9,3 & 5,0 & 3,50 & 5,8 & 3,8 & 4,0 & 0,5 & 2,13 & $0,03^{*}$ \\
\hline FA & 8,3 & 4,5 & 7,5 & 4,75 & 8,1 & 4,8 & 6,5 & 6,0 & 0,52 & 0,60 \\
\hline
\end{tabular}

BEP = bem-estar positivo; DPS = distress psicológico; FA = fadiga; DP= desvio-padrão; DI= desvio interquartílico.

*Diferença estatística significativa. 
Tabela 3. Média, desvio-padrão, mediana, desvio interquartílico e resultados do teste Wilcoxon para cada subescala da SEES referentes ao exercício realizado em $\mathrm{AV}(\mathrm{n}=20)$.

\begin{tabular}{lcccccccccc}
\hline & \multicolumn{9}{c}{ Antes } & \multicolumn{3}{c}{ Depois } \\
\hline SEES & Média & DP & Mediana & DI & Média & DP & Mediana & DI & z & $p$ \\
BEP & 20,1 & 5,8 & 21,0 & 11,25 & 23,5 & 4,1 & 24,0 & 6,0 & 2,45 & $0,01^{*}$ \\
DPS & 6,7 & 3,0 & 6,0 & 4,0 & 4,9 & 1,9 & 4,0 & 1,0 & 2,11 & $0,03 *$ \\
FA & 8,85 & 5,2 & 8,5 & 8,0 & 6,65 & 3,9 & 5,5 & 3,25 & 1,87 & 0,06 \\
\hline
\end{tabular}

BEP = bem-estar positivo; DPS = distress psicológico; FA = fadiga; DP = desvio-padrão; DI= desvio interquartílico.

*Diferença estatística significativa.

Tabela 4. Resultados do teste Mann-Whitney para cada subescala da SEES comparando o exercício realizado em AR com o realizado em $\mathrm{AV}(\mathrm{n}=20)$.

\begin{tabular}{lcc}
\hline Bem-estar positivo & $\mathrm{U}$ & $p$ \\
Antes & 143 & 0,12 \\
Depois & 188 & 0,74 \\
& & \\
Distress psicológico & 182,5 & 0,63 \\
Antes & 185 & 0,68 \\
Depois & & \\
& & \\
Fadiga & 195 & 0,89 \\
Antes & 173,5 & 0,47 \\
Depois & & \\
\hline
\end{tabular}

curta, em uma amostra de 291 idosos frequentadores de centros de convivência, foi verificado que 38,1\% estavam classificados no nível "alto", 49,8\% no nível "moderado" e 12,1\% no nível "baixo" de atividade física.

Em relação às experiências subjetivas relacionadas ao exercício, foi observado no presente estudo aumento no bemestar positivo das idosas após a realização do exercício de dança em ambiente virtual. Outros estudos também encontraram resultados positivos em relação ao aumento do bem-estar positivo após a prática de exercício. Bartholomew, Morrison, e Ciccolo (2005) realizaram um estudo com 40 indivíduos que estavam recebendo tratamento para transtorno depressivo. Os voluntários foram divididos em dois grupos. Um dos grupos executou 30 minutos de caminhada em esteira a 60/70\% da frequência cardíaca máxima predita para a idade, enquanto que o outro grupo descansou tranquilamente durante o mesmo período de tempo. Os participantes responderam à SEES 5 minutos antes e, 5, 30 e 60 minutos após as sessões experimentais. Foi encontrado que apenas o grupo que realizou o exercício em esteira apresentou um aumento significativo no bem-estar positivo.

No estudo de Cox, Thomas, e Davis (2001), que tinha como objetivos estudar, além da variação dos afetos positivos e negativos associados à intensidade e ao modo do exercício, a duração desses efeitos após a prática de uma sessão aguda de exercício, foi observado o aumento do bemestar positivo logo após a realização da atividade. Este aspecto, ou seja, o aumento do bem-estar positivo continuou aumentando nos próximos 30 e 60 minutos após o término do exercício aeróbio.
Embora no ambiente virtual a sensação de fadiga tenha apresentado uma tendência à redução logo após o exercício nesse ambiente, no presente estudo não foram observadas alterações estatisticamente significativas na sensação de fadiga após as sessões em ambos os ambientes de exercício. Contudo, diversos resultados são evidenciados em outros estudos encontrados na literatura. Sealy (2010) conduziu um experimento com a finalidade de comparar a experiência subjetiva em três diferentes combinações de exercícios realizados em sessão aguda, com homens idosos sedentários que responderam a SEES antes e depois da realização de cada combinação de exercício. O grupo que participou da combinação com maior número de exercícios reportou um ligeiro aumento do distress psicológico e o maior aumento da fadiga. Outros pesquisadores encontraram diferentes resultados a respeito da sensação de fadiga após exercício, tais como: nenhuma alteração (Jerome, Marquez, McAuley, Canaklisova, Snook, \& Vickers, 2002), aumento (Cox et al., 2001; Watt \& Spinks, 1997) e redução deste parâmetro (Daley \& Welch, 2004).

Foi possível perceber no presente estudo que houve redução do distress psicológico após a prática do exercício em ambas as situações experimentais (ambientes real e virtual). Cox et al. (2001) também encontraram diminuição do distress psicológico após 30 e 60 minutos da prática de exercícios aeróbios (esteira e stepping). Na mesma direção, Daley e Huffen (2003) evidenciam que a prática de exercício aeróbio pode contribuir para a redução do distress psicológico e da fadiga.

A maioria das idosas participantes do estudo relatou ter experiência anterior em algum tipo de dança e/ou ginástica. A escolha do exercício físico, com uma versão equivalente em jogos ativos que seria utilizado no presente estudo, se pautou nos benefícios dos exercícios de dança para a população idosa (Barreto \& Sanches, 2011). De acordo com o estudo de revisão realizado por Keogh, Kilding, Pidgeon, Ashley, e Hillis (2009), a dança é uma forma de atividade física que resulta em benefícios físicos que podem levar idosos a melhorar sua saúde e bem-estar. A referida revisão incluiu artigos cujas amostras eram compostas por indivíduos saudáveis com 60 anos ou mais e que permitiam a comparação de praticantes de dança (pelo menos oito semanas de participação) com não praticantes. A partir dos resultados dos estudos encontrados (15 estudos 
experimentais e três estudos transversais), os autores verificaram que um programa de exercício baseado em atividades de dança aprimora a potência aeróbia, a resistência muscular, a força e flexibilidade de membros inferiores, equilíbrio estático e dinâmico e agilidade, bem como a velocidade de marcha (Keogh et al., 2009).

A maioria das idosas pesquisadas relatou preferência pela prática de exercício em ambiente real. Esse resultado pode estar associado ao fato de todas elas terem relatado que nunca haviam jogado nenhum tipo de videogame anteriormente, o que pode ter influenciado na escolha do ambiente real como o preferido. Essas idosas se depararam com uma experiência corporal nova, anteriormente não vivenciada.

Embora não tenham sido encontrados estudos na literatura que relacionam a questão da preferência entre o ambiente real e o virtual para a prática de exercício em idosos, há evidências da existência de fundamentos para se considerar que o exercício em ambiente virtual pode, além de propiciar momentos de entretenimento de grande qualidade, levar a alterações positivas em aspectos sociais e emocionais. Allaire et al. (2013) buscaram investigar se havia diferenças socioemocionais (bem-estar, afeto, depressão, funcionamento social) entre idosos praticantes de jogos digitais comparados a idosos não praticantes. Este estudo transversal contou com 140 idosos de ambos os sexos que foram divididos em três grupos distintos: jogadores regulares, jogadores ocasionais e não jogadores. Os jogadores regulares e ocasionais apresentaram melhor desempenho quando comparados aos idosos não praticantes, em relação às variáveis pesquisadas. Assim, os resultados desta pesquisa sugerem que os jogos digitais podem ser considerados uma atividade positiva associada ao envelhecimento bem sucedido.

Ainda com relação aos aspectos psicológicos, um estudo realizado na Universidade da Califórnia, nos Estados Unidos, revelou que os videogames ativos podem diminuir bastante os sintomas da Depressão Subsindrômica (SSD) em pessoas idosas. O termo Depressão Subsindrômica tem sido usado na literatura para designar um conjunto de sintomas depressivos que não preenchem completamente o quadro de depressão, já que não há sintomas suficientes e intensos, mas que implica algum grau de sofrimento para as pessoas afetadas (Rosemberg et al., 2010).

Dessa forma, os videogames ativos além de proporcionar melhoras em diferentes fatores psicológicos, têm se apresentado para o idoso como uma boa opção para o tempo de lazer e como mecanismo de auxílio para a prática de exercícios físicos. De acordo com Schiesel (2007), essa população tem demonstrado grande receptividade às novas tecnologias de informação, optando pelos videogames como atividade física quando se busca a descontração e o entretenimento.

Apesar de indícios encontrados na literatura que demonstram o aumento gradativo do interesse de pessoas idosas pelo uso de novas tecnologias (Schiesel, 2007), como os jogos eletrônicos, muitos idosos ainda não estão habituados com a ideia da utilização de um videogame para a prática de exercício. Isso pode ser devido ao fato de muitos idosos terem convivido por muito tempo com a ideia negativa de que videogames apenas eram utilizados por jovens que permaneciam sentados em frente à TV, se entretendo com os jogos tradicionais, ou seja, aqueles em que os movimentos se restringem apenas a manipulação do controle, por um grande período de tempo. Na opinião de Papastergiou (2009), a utilização dos videogames foi vista por muitos anos como uma atividade que poderia resultar em diversos problemas para a saúde como, por exemplo, alguns tipos de lesões, comportamento agressivo e tempo reduzido para conviver com outras pessoas.

A baixa preferência das idosas pelo ambiente virtual para a prática de exercício pode remeter à discussão da questão geracional. De acordo com Garcia (2001), os idosos, quando jovens, viveram em um período em que atitudes e valores eram adotados diferentemente dos que são incorporados hoje em dia. Atualmente, atividades cotidianas são constantemente mediadas por novas tecnologias, tais como, o uso de computador, internet, telefones celulares e jogos eletrônicos. Sendo assim, essa transformação rápida e complexa das mudanças tecnológicas que afetam a maneira como as pessoas se relacionam pode representar para o idoso uma nova mudança de atitude. Em consequência, pode ser ocasionado certo tipo de dificuldade e até resistência em relação à apropriação das novas tecnologias por essa população. Geralmente as pessoas demoram algum tempo para incorporar em suas vidas os elementos das novas tecnologias, uma vez que o ser humano demonstra certa tendência a resistir às mudanças, temendo as novidades e as situações desconhecidas (Garcia, 2001).

\section{Considerações finais}

A partir dos resultados do atual estudo pode-se perceber que a prática de exercício, tanto em ambiente real, quanto virtual, é benéfica para as pessoas idosas, no que tange aos aspectos referentes à percepção subjetiva relacionada ao exercício. Foi possível constatar que o uso de jogos eletrônicos classificados como videogames ativos torna-se efetivo para a população idosa, quando se visa alcançar resultados positivos relacionados às respostas psicológicas associadas com a participação em um exercício agudo.

Apesar de as relações entre videogame e prática de atividades físicas e/ou sedentarismo estarem em processo de investigação mais aprofundada atualmente na literatura, a chegada dos jogos eletrônicos, que permitem maior interação corporal, traz a ideia de uma nova tendência de destacar a propensão "ativa” dos videogames com ênfase na execução do jogo, utilizando-se dos movimentos do próprio corpo.

Novas pesquisas poderiam ser conduzidas explorando outros elementos que pudessem influenciar nas respostas psicológicas associadas ao exercício como, por exemplo, sessão de exercício com maior duração pela utilização de uma música a mais ou a repetição seguida da mesma música em cada sessão; a utilização de outros jogos que também 
simulem situações reais; e a comparação entre amostras de indivíduos que apresentam diferentes características como faixa de idade, experiência com videogames, entre outras. Tornam-se relevantes novos estudos, no sentido de compreender outros aspectos referentes aos estados subjetivos envolvidos nas atividades físicas, ampliando as reflexões sobre as complexas relações entre o idoso, o ambiente virtual e a prática de exercícios físicos.

\section{Referências}

Allaire, J. C., McLaughlin, A. C., Trujillo, A., Whitlock, L. A., LaPorte, L., \& Gangy, M. (2013). Successful aging through digital games: Socioemotional differences between older adult gamers and Non-gamers. Computers and Human Behavior, 29(4), 1302-1306. Retrieved from http://ww.sciencedirect.com/ science/article/pii/S0747563213000174

Ayres, M., Ayres Júnior, M., Ayres, D. L., \& Santos, A. A. S. (2007). BioEstat 5.0.: aplicações estatísticas nas áreas das Ciências Biomédicas. Belém: Ong Mamiraua.

Baracho, A. F. O., Gripp, F. J., \& Lima, M. R. (2012). Os exergames e a Educação Física Escolar na cultura digital. Revista Brasileira de Ciências do Esporte, 34(1), 111-126. Retrieved from http:/ /www.rbceonline.org.br/revista/index.php/RBCE/article/view/ $1017 / 725$

Barreto, P. S., \& Sanches, J. C. J. (2011). Long-term adherence to exercise: The relationship with functional fitness and personal motivation among community-dwelling independent-living older women. Revista Brasileira de Ciências do Esporte, 33(1), 193-206. Retrieved from http://www.scielo.br/ scielo.php?pid=S0101-32892011000100013\&scrip $\mathrm{t}=$ sci_arttext

Bartholomew, J. B., Morrison, D., \& Ciccolo, J. T. (2005). Effects of Acute Exercise on Mood and Well-Being in Patients with Major Depressive Disorder. Medicine e Science in Sports Exercise, 37(12), 2032-2037. Retrieved from http:// www.ncbi.nlm.nih.gov/pubmed/16331126

Benedetti, T. R. B., Binotto, M. A., Petroski, L., \& Gonçalves, L. H. T. (2008). Atividade física e prevalência de quedas em idosos residentes no sul do Brasil. Revista Brasileira de Geriatria e Gerontologia, 11(2), 1-13. Retrieved from http:// revista.unati.uerj.br/scielo.php ?script =s ci_ arttext\&pid=S1809-98232008000200002\&lng=pt\&nrm=iso

Berger, B. G., \& Motl, R. W. (2000). Exercise and mood: A selective review and synthesis of research employing the profile of mood states. Journal of Applied Sport Psychology, 12(1), 69-92. Retrieved from http://www.tandfonline.com/doi/abs/10.1080/ 10413200008404214

Cox, R. H., Thomas, T. R., \& Davis, J. E. (2001). Positive and Negative Affect associated with and acute bout of aerobic exercise. Journal of Exercise Physicologic, 4(4), 13-20 Retrieved from http://programmingz.com/b/554356/Effects-Of-ExerciseOn-Mood-Jep-Online-Journal-Of-Exercise-/\#.UVDfvhzFVqU

Daley, A. J., \& Huffen, C. (2003). The Effects of Low and Moderate Intensity Exercise on Subjective Experiences in a Naturalistic Health and Fitness Club Setting. Journal of Health Psychology, 8(6), 685-691. Retrieved from http:// www.ncbi.nlm.nih.gov/pubmed/14670203

Daley, A., \& Welch, A. (2004). The effects of $15 \mathrm{~min}$ and $30 \mathrm{~min}$ of exercise on affective responses both during and after exercise. Journal of Sports Sciences, 22(7), 621-628. Retrieved from http://www.ncbi.nlm.nih.gov/pubmed/15370492
Focht, C. B., Knapp, J. B., Gavin, P. T., Raedeke, D. T., \& Hickner, C. R. (2007). Affective and Self-Efficacy Responses to Acute Aerobic Exercise in Sedentary Older and Younger Adults. Journal of Aging and Physical Activity, 15(2), 123-138. Retrieved from http://www.ncbi.nlm.nih.gov/pubmed/ 17556780

Garcia, H. D. (2001). A terceira idade e a Internet: uma questão para o novo milênio. (Dissertação de Mestrado, Universidade Estadual Paulista, Marília). Retrieved from http:// www.marilia.unesp.br/Home/Pos-Graduaca o/ CienciadaInformacao/Dissertacoes/dominguez_ garcia_me_mar.pdf

Guidelines for Data Processing and Analysis of the International Physical Activity Questionnaire (IPAQ) - Short and long forms. (2005). Retrieved from http://www.ipaq.ki.se/scoring.pdf

Haddock, B. L., Jarvis, S., Klug, N. R., Gonzalez, T., Barsaga, B., Siegel, S. R., \& Wilkin, L. D. (2012). Measurement of Energy Expenditure while Playing Exergames at a Selfselected Intensity. The Open Sports Sciences Journal, 5(2012), 1-6. Retrieved from http://bentham science.com/open/tossj/articles/V005/ 1TOSSJ.pdf

Hallal, P. C., Victoria, C. G., Wells, J. C., \& Lima, R. C. (2003) Physical inactivity: prevalence and associated variables in brazilian adults. Medicine and Science in Sports and Exercise, 35(11), 1894-1900. Retrieved from http:// www.ncbi.nlm.nih.gov/pubmed/14600556

Hoffman, S. J., \& Harris, J. C. (2002). Cinesiologia: O Estudo da Atividade Física (3a Ed). Porto Alegre: Artmed.

Jerome, J. S., Marquez, D. X., McAuley, E., Canaklisova, S., Snook, E., \& Vickers, M. (2002). Self- efficacy effects on feeling states in women. International Journal of Behavioral Medicine, 9(2), 139-154. Retrieved from http://www.ncbi.nlm.nih.gov/ pubmed/12174532

Keogh, J. W. L, Kilding, A., Pidgeon, P., Ashley, L., \& Gillis, D. (2009). Physical Benefits of Dancing for Healthy Older Adults: A Review. Journal of Aging and Physical Activity, 17(4), 479500. Retrieved from http://www.ncbi. nlm.nih.gov/pubmed/ 19940326

Krug, R. R., Conceição, J. C. R., Mazo, G. Z., Antunes, G. A., \& Romitti, J. C. (2011). Contribuições da caminhada como atividade física de lazer para idosos. Licere, 14 (4), 1-29. Retrieved from http://www.anima.eefd.ufrj.br/licere/pdf/ licereV14N04_a3.pdf

Matsudo, S. M., Matsudo, V. R., Araújo, T., Andrade, D., Andrade, E., \& Oliveira, L. (2002). Nível de atividade física da população do estado de São Paulo: análise de acordo com o gênero, idade, nível socioeconômico, distribuição geográfica e de conhecimento. Revista Brasileira de Ciência e Movimento, 10(4), 41-50. Retrieved from http://portalrevistas.ucb.br/ index.php/RBCM/article/view/469

McAuley, E., \& Courneya, K. S. (1994). The Subjective Exercise Experiences Scale (SEES): Development and Preliminary Validation. Journal of Sports and Exercise Psychology, 16(2), 163-177. Retrieved from http://www.epl.illinois.edu/files/ images/measures/SEES_article.pdf

McAuley, E., Elsavsky,.S., Jerome, G. J., Konopack, J. F., \& Marquez, D. X. (2005). Physical activity related well being in older adults: social cognitive influences. Psychology and Aging, 20(2), 295-302. Retrieved from http://www.ncbi.nlm.nih.gov/ pubmed/16029093

Miranda, M. L. (2001). Efeitos da atividade física com música sobre estados subjetivos de idosos (Tese de Doutorado). Universidade de São Paulo, São Paulo, SP. 
Nelson, M. E. W. J., Rejeski, S. N., Blair, P. W., Duncan. J. O., Judge, A. C., King, C. A., Macera, \& Castanedasceppa, C. (2007) Physical Activity and Public Health in Older Adults: Recommendation from the American College of Sports Medicine and the American Heart Association. Medicine and Science of Sports and Exercise, 39(8), 1435-1445.

Papastergiou, M. (2009). Exploring the potential of computer and video games for health and physical education: A literature review. Computers and Education, 53(3), 603-622. Retrieved from http://dx.doi.org/10.1016/j.compedu.2009.04.001

Rosemberg, D., Depp, C. A., Vahia, I. V., Reichstadt, J., Palmer, B. W., Kerr, J., Norman, G., \& Jeste, D. V. (2010). Exergames for Subsyndromal Depression in Older Adults: A Pilot Study of a Novel Intervention. The American Journal of Geriatric Psychiatry, 18(3), 221-226. Retrieved from http:// www.ncbi.nlm.nih.gov/pubmed/20173423

Schiesel, S. (2007, March 30). Video Games Conquer Retirees. New York Times, Retrieved from http://www.nytimes.com/ 2007/03/30/arts/30seni.html?pagewanted=all\&_r=0

Sciolino, N. R.; \& Holmes, P. V. (2012). Exercise offers anxiolytic potential: A role for stress and brain noradrenergic-galaninergic mechanisms. Neuroscience and Behavioral Reviews, 36(9), 1965-1984. Retrieved from http://dx.doi.org/10.1016/ j.neubiorev.2012.06.005

Sealy, R. M. (2010). Acute Exercise inVietnan Veterans in Associated with Positive Subjetive Experiences. International Journal of Exercise Science, 3(1), 36- 42. Retrieved from http:/ / d i g i t a l c o m m o n s. w k u e d u / c g i / viewcontent.cgi?article $=1200 \&$ context $=$ ijes

Siegel, S. (Ed. 1). (1975). Estatística não-paramétrica para Ciências do Comportamento. São Paulo: McGraw-Hill.

Siqueira, F. V., Facchini, L.A., Tomasi, E., Thumé, E., Silveira, D. S., Vieira,V., \& Hallal, P.C. (2007). Prevalência de quedas em idosos e fatores associados. Revista Saúde Pública, 41(5), 749756. Retrieved from http://dx.doi.org/10.1590/S003489102007000500009

Tanaka, K., Parker, J., Baradoy, G., Sheehan, D., Holash, J. R., \& Katz, L. A. (2012). Comparison of Exergaming Interfaces for Use in Rehabilitation Programs and Research. Loading... The Journal of the Canadian Game Studies Association, 6(9), 6981. Retieved from http://journals.sfu.ca/loading/index.php/ loading/article/view/107/118

Thomas, J. R., \& Nelson, J. K. (Ed. 3). (2002). Métodos de pesquisa em atividade Física. Porto Alegre: Artmed.

Torres, A., \& Zagalo, N. (2007). Videojogos: um novo meio de entretenimento de idosos. Artigo apresentado no $5^{\circ}$ Congresso da Associação Portuguesa de Comunicação Braga, Braga. Retrieved from http://www.lasics.uminho.pt/ojs/index.php/ 5sopcom/article/view/191/215

Vaghetti, C. A. O., \& Botelho, S. S. C. (2010). Ambientes virtuais de aprendizagem na Educação Física: uma revisão sobre a utilização de Exergames. Ciências e Cognição, 15(1), 76-88. Retrieved from http://www.cienciasec ognicao.org/revista/ index.php/cec/article/view/292/162

Valim-Rogatto, P. C., Candolo, C., \& Brêtas, A. C. P. (2011). Nível de atividade física e a sua relação com quedas acidentais e fatores psicossociais em idosos de centro de convivência. Revista Brasileira de Geriatria e Gerontologia, 14(3), 521533. Retrieved from http://www.scielo.br/pdf/rbgg/v14n3/ v14n3a12.pdf

Watt, B. J., \& Spinks, W. L. (1997). Dynamics of exercise induced affect. Journal of Sports Medicine, 29(3), 69-74. Retrieved from http://www.ncbi.nlm.nih.gov/pubmed/9302489
Wii Consoles. Wii Hardware Specification. (2009). Retrieved from www.wiiconsoles.co.uk/wiihardwarespecifica tion.asp

Williams, M. A., Soiza, R. L., Jenkinson, A. M., \& Stewart, A. (2010). Exercising with Computers in Later Life (EXCELL) pilot and feasibility study of the acceptability of the Nintendo WiiFit in community-dwelling fallers. BMC Research Notes, 13(3), 2- 8. Retrieved from http://www.biomedcentral.com/ $1756-0500 / 3 / 238$

WHO (World Health Organization). (1995). Physical status: the use and interpretation of anthropometry. Geneva: World Health Organization.

\section{Nota dos autores}

Amanda Mayara do Nascimento, Nara Heloisa Rodrigues e Gisele Maria Schwartz são filiadas ao Laboratório de Estudos do Lazer, Universidade Estadual Paulista (UNESP).

Eric Francelino Andrade e Gustavo Puggina Rogatto são filiados ao Laboratório de Investigação e Estudos sobre Metabolismo e Exercício Físico, Universidade Federal de Lavras (UFLA).

Priscila Carneiro Valim-Rogatto é filiada ao Laboratório Pesquisa em Psicologia do Exercício, Universidade Federal de Lavras (UFLA)

\section{Correspondência}

Universidade Federal de Lavras, Departamento de Educação Física, Campus Universitário

Caixa Postal 37 Lavras, MG. Brasil.

Telefones: 3538295136 ou 3538291293

Email: amandinhamn@yahoo.com.br

Artigo completo de trabalho apresentado no VIII Congresso Internacional de Educação Física e Motricidade Humana e XIV Simpósio Paulista de Educação Física.

Declaração de Conflito de Interesses: Os autores declararam ausência de conflitos de interesse no que diz respeito à pesquisa, autoria e/ou publicação deste artigo.

Manuscrito recebido em 30 de março de 2013

Manuscrito aceito em 10 de maio de 2013 